\title{
Modelling the permeability of polymers: a neural network approach
}

\author{
M. Wessling, ${ }^{a, 1}$, M.H.V. Mulder ${ }^{a, *}$, A. Bos ${ }^{b}$, M. van der $\operatorname{Linden}^{b}$, M. Bos ${ }^{b}$ and W.E. van der \\ Linden ${ }^{\mathrm{b}}$ \\ Membrane Technology and ' ${ }^{-}$Chemical Analysis, Faculty of Chemical Technology, University of Twente, P.O. Box 217, \\ 7500 AE Enschede (Netherlands)
}

(Received July 16, 1993; accepted in revised form September 25, 1993)

\begin{abstract}
In this short communication, the prediction of the permeability of carbon dioxide through different polymers using a neural network is studied. A neural network is a numeric-mathematical construction that can model complex non-linear relationships. Here it is used to correlate the IR spectrum of a polymer to its permeability. The underlying assumption is that the chemical information hidden in the IR spectrum is sufficient for the prediction. The best neural network investigated so far does indeed show predictive capabilities.
\end{abstract}

Key words: gas and vapor permeation; theory

\section{Introduction}

Mass transport properties of polymer films are of special interest in the food packaging industry and in membrane technology [1]. The most important property of a polymeric material for these applications is the permeability, i.e., the pressure- and thickness-normalized flux through the polymer film. The permeability of a gas through a polymer film depends on the physico-chemical characteristics of the gas and

${ }^{1}$ Current address: MTR, 1360 Willow Road 103, Menlo Park, CA 94303, USA.

*To whome correspondence should be addressed. the polymer. In this communication we will focus only on the influence of polymer properties on the permeability of carbon dioxide as the permeating gas.

A considerable amount of research effort is spent on the correlation of mass transport properties of polymers having different chemical structures. A widely used and accepted theory is the correlation between the permeability and the free volume of the polymer [2]. However, this correlation does not hold strictly for all polymers [3,4]. Another correlation method is the Permachor method [5] based on group contribution. In this method, a certain numerical value is coupled to a specific chemical group 
present in the polymer repeat unit. The sum of these values over all different groups present in the polymer is linearly related to the permeability of a certain gas.

In our approach, we do not distinguish between certain chemical groups based on the chemical formula. Instead, it is assumed that the information hidden in the IR spectrum of a polymer is sufficient to provide a correlation with the permeability of the polymer. This assumption may be debatable, however, a number of publications described below make the idea acceptable. Koros and Fleming [6] describe the extraction of general rules from permeation experiments with chemically different polymers, relating polymer backbone modifications to alterations in mass transport characteristics. Yampolski [7], on one hand, relates the permeability to the glass transition temperature, and Hopfinger et al. [8], on the other hand, relate the glass transition temperature to the chemical structure of the polymer. The chemical structure of a polymer, finally, is sufficiently characterized by its IR spectrum. However, the relationship between the IR spectrum of a polymer and its permeability is not obvious and this short communication is only a first attempt to answer the question on the existence of such a relationship.

The attempt to correlate IR spectra to the permeability is not completely new. In a U.S. patent, Gold [9] correlates the permeability of gases through a mixture of two epoxy resins with their IR spectra. However, the mathematical tool used (Partial Least Square Regression) is probably not powerful enough for different polymers which may vary considerably in chemical structure.

\section{Neural networks}

In this study, a neural network was chosen as a mathematical tool to correlate the IR spectrum to the permeability of the polymer. In neural network science, the objective is to mimic mathematically the processing of input signals to an output signal as in signal processing in biological neural systems [10]. Because of the non-linearity of the neural networks, this approach is very powerful in correlating complex input signals to output signals that are in some way related. Here, the complex IR spectrum is the input signal and the permeability the output signal. Before focusing on the methodology of the study, the principle of neural networks is briefly explained.

A neural network consists of a network of processing elements, so-called "neurons". These neurons are organized in layers in the network as shown in Fig. 1a. Every neuron calculates an output $o_{i}$ out of all incoming inputs $x_{i}$. Generally, for a neuron $i$, all inputs $x_{j}$ are weighted by a factor $w_{i j}$ and then summed up to the final input $u_{i}$.

$u_{i}=\sum_{j} w_{i j} x_{j}$

This input can be transferred into the output $o_{i}$ by a mathematical transfer or "squashing" function. Different kinds of neurons can have different transfer functions: a sigmoid neuron transfers the input into the output according to eqn. (2) with $\theta$ as a bias.

$o_{i}=\frac{1}{1+\exp \left(-u_{i}+\theta\right)}$

On the other hand, a neuron having a radial base function transfers directly all inputs $x_{j}$ into an output according to:

$$
\begin{aligned}
& o_{i}=\exp \left(\frac{\sum_{j}\left(x_{j}-w_{i j}\right)^{2}}{-2 \sigma_{i}^{2}}\right) \\
&=\prod_{j} \exp \left(\frac{\left(x_{j}-w_{i j}\right)^{2}}{-2 \sigma_{i}^{2}}\right)
\end{aligned}
$$

The correct input-output relation of the network as a whole is "learned" from examples by adapting the weights and the additional pa- 

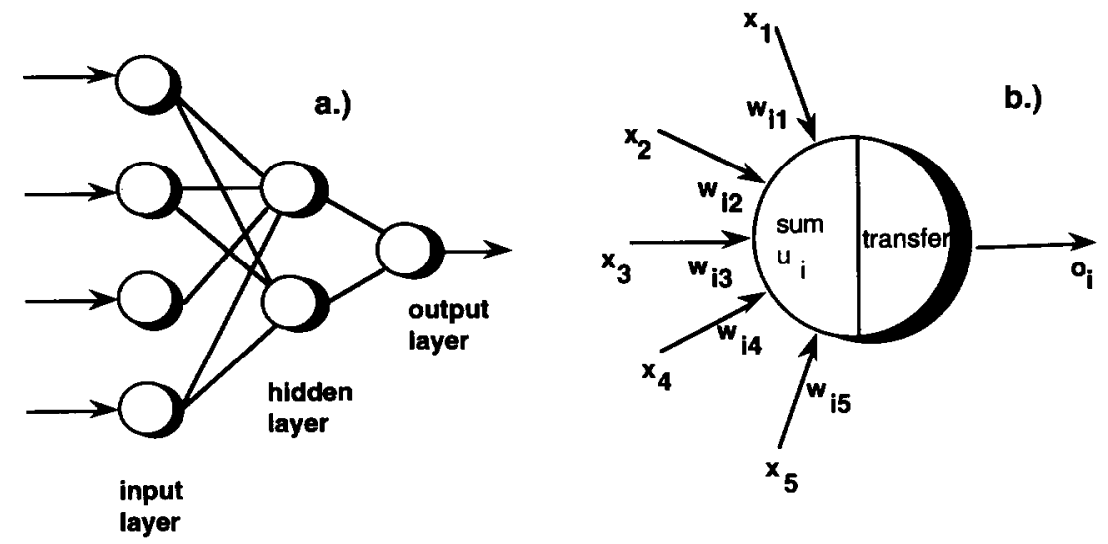

Fig. 1. Schematic drawing of a neural network topology (a) and a processing element called neuron (b).

rameters $\sigma_{i}$ and $\theta_{i}$ iteratively. To develop an optimal network that correlates the permeability of a polymer to its IR spectrum, a number of network parameters can be changed. A network with an optimal topology must be found with respect to the kind of neuron used, the number of layers and the number of neurons per layer.

First an adequate number of neurons in the input layer is chosen. Secondly, a certain set of IR spectra together with the permeabilities is presented to the network. (The method of presenting the IR spectrum to the input layer will be explained later.) The third step is to train the network by changing the weights of neurons in such a way that a minimum error is obtained between the predicted and experimental permeability. Training and testing was carried out in this study according to the leave-one-out method. One polymer is left out of the set of polymers and, after the network has been trained with the remaining polymers, the permeability of this polymer is predicted on the basis of its IR spectrum. The procedure is repeated for all polymers in the training set.

In order to measure the predictive power of different network topologies, a performance factor, $C$, has been defined [see eqn. (4)]. This performance factor, $C$, is equal to the ratio of the sum of all differences between the predicted output (output) and the experimental permeability (target output) and the sum of all differences between the average permeability $P_{\mathrm{av}}$ of all polymers and the experimental permeability.

$C=\frac{\sum_{n} \mid \text { output }- \text { target output } \mid}{\sum_{n} \mid P_{a v}-\text { target output } \mid}$

The neural network works predictively if $C$ is smaller than 1 . For $C=1$, the predicted permeability for an unknown polymer would be equal to the average permeability of all polymers presented in the set (which is, in fact, useless).

\section{Infrared spectroscopy}

The question that remains is how an IR spectrum, which is rather complex in nature, can be presented as a valuable, compact input to the neural network. We have chosen a curve-fitting procedure using Legendre polynomials [11]. The IR spectra were fitted in the range 400 $2000 \mathrm{~cm}^{-1}$. Depending on the number of polynomials used, the quality of the representation of the IR spectra can be influenced, as shown 


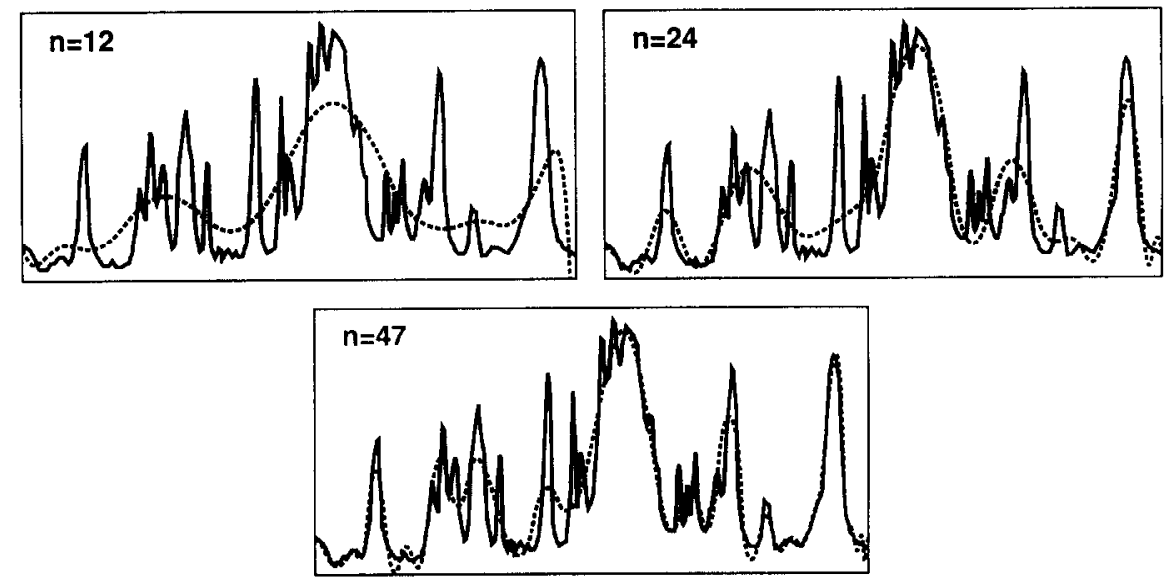

Fig. 2. Comparison of fitted and experimental IR spectra for bisphenol-A polycarbonate at different numbers of Legendre polynomials.

in Fig. 2. A maximum set of 48 polynomials, resulting in 48 input values for the network, could be processed by the network. This, however, does not automatically imply an optimum input layer topology. Although 48 polynomials was not sufficient to fit every peak in the spectrum, the general form of the spectrum was reproduced well by this method.

\section{Set of polymers}

Not only the quality of inputs and the topology of networks, but also the quality of the polymer set itself may strongly influence the success of neural network modelling. An optimum polymer set would comprise at least 50 different polymers (the more the better) with a very subtle change in polymer structure from one polymer family to another. The polymers used in this study were all glassy polymers. In this study, 33 glassy polymers were used for the modeling. The set of polymers consisted of tailor-made polyimides [12], polycarbonates [13], cellulosic polymers, polysulfones and aromatic polyesters. Polymers were dissolved in suitable solvents, cast on glass plates, dried in a nitrogen atmosphere for $24 \mathrm{~h}$ and finally dried for 2 weeks in a vacuum oven at $70^{\circ} \mathrm{C}$. Permeabili- ties were measured at $32^{\circ} \mathrm{C}$ using a set-up described in [12].

\section{Results and discussion}

A considerable amount of effort and computing time was invested in the optimization of the network topology details [14]. Besides the fitting of the Legendre polynomials, other techniques were also used and compared. Only the best results obtained to date are presented below. These were obtained with a neural network with 24 input neurons and 16 radial base function neurons in a single hidden layer. The IR spectrum was fitted using 47 polynomials, but only the first 24 were presented to the network. This procedure is valid due to the orthogonality of Legendre polynomials. The network was trained in 3000 iterations for each polymer.

Figure 3 shows the predicted permeability for each polymer versus the experimental permeability. Both the $y$ and $x$ axes are logarithmic, because a linear axis would cluster most of the data points in the range 1-20 barrer ( 1 barrer $=10^{-10} \mathrm{~cm}^{3}$ (STP) $\mathrm{cm} /\left(\mathrm{cm}^{2} \mathrm{~s} \mathrm{cmHg}\right)$. The solid line represents equal values for the predicted and experimental permeability.

A clear correlation between the predicted and 


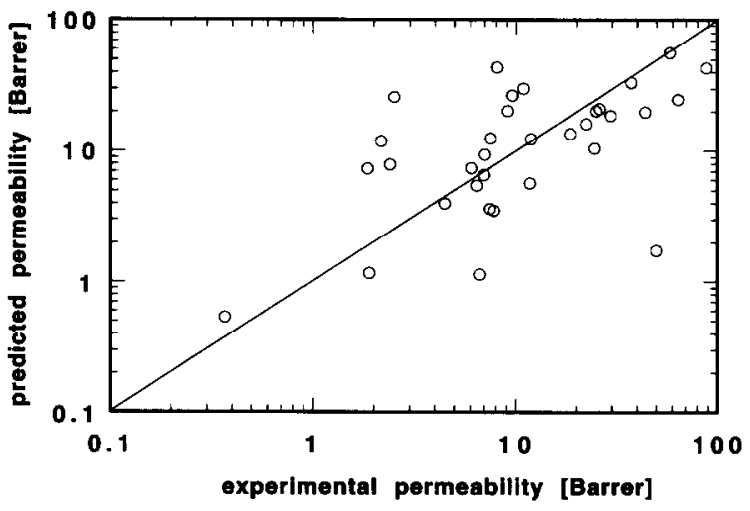

Fig. 3. Comparison of the permeabilities predicted by the neural network and the experimental permeability of carbon dioxide in different polymers.

the experimental permeability can be seen. The correlation is also expressed by the performance factor of $C=0.77$. Extreme values for the lowest and highest permeability must be extrapolated by the network because these polymers are not present in the training set. Satisfactory agreement between predicted and experimental permeabilities was obtained in the case of phenoxy resin at 0.5 barrer and poly $(2,6-$ diphenyl-1,4-phenylenoxide) at 100 barrer, respectively.

On the other hand, some polymers do not fit well on the line. In general, these are polymers containing certain groups which occur only once in the whole set of polymers. Thus, if they are taken out during the leave-one-out training, the network is trained without this group. In the case of the polymer poly (vinyl butyrate), the experimental permeability of 8.1 barrer is significantly smaller than the predicted value of 43.8 barrer. PIXU 218 from Ciba Geigy deviates in the other direction: the experimental permeability of 50 barrer is completely underestimated by the prediction of 1.7 barrer.

In summary, this short communication shows that neural networks are able to relate the IR spectrum of a polymer to its permeability for carbon dioxide. Enlargement of the polymer set can be expected to improve the quality of the prediction.

In using this particular correlation methodology, it is important to note that the weights of the final trained network do not have any physical significance. Only a systematic artificial manipulation of an input IR spectrum, representing an alteration in the chemical structure of the polymer, together with the newly predicted permeability may give more insight into the molecular aspects influencing the permeability. It is not clear yet whether history effects like annealing or residual solvent effects can be included in the modelling. The authors are aware of the impact of such effects on the permeability. On the other hand, the extent of crystallization as well as the residual solvent content can be determined by IR spectroscopy. Certainly, this neural network method can also be applied to mechanical and thermal properties of polymers such as the elastic modulus or the glass transition temperature, respectively.

\section{References}

1 W.J. Koros, Barrier polymers and structures, ACS Symp. Ser., 423 (1989) 1.

2 W.M. Lee, Selection of barrier material from molecular structure, Polym. Eng. Sci., 20 (1980) 65.

3 E. Hensema, M.H.V. Mulder and C.A. Smolders, On the mechanism of gas transport in rigid polymer membranes, J. Appl. Polym. Sci., 49 (1993) 2081.

4 M. Wessling, T. van den Boomgaard, M.H.V. Mulder and C.A. Smolders, Transport of gases through polymeric membranes, Makromol. Chem., Macromol. Symp., 70/71 (1993) 379.

5 D.W. van Krevelen, Properties of Polymers, 3rd edn., Elsevier, Amsterdam, 1990.

6 W.J. Koros and G.K. Fleming, Membrane-based gas separation, J. Membrane Sci., 83 (1993) 1.

7 Y.P. Yampolski, Sorption and gas and vapor permeability in membranes based on glassy polymers. Role of free volume, in A.M. Mika and T.Z. Winnicki (Eds.), Advances in Membrane Phenomena and Processes, Wrocław Techn. Univ. Press, Wrocław, 1989.

8 A.J. Hopfinger, M.G. Koehler and R.A. Pearlstein, Molecular modeling of polymers. IV. Estimation of glass transition temperatures, J. Polym. Sci., Polym. Phys., 26 (1988) 2007. 
9 H.S. Gold, U.S. Pat. 5.015 .856 (1991).

10 D.E. Rumelhardt, Parallel Distributed Processing, Vol. 1 and 2, MIT Press, Cambridge, MA, 1986.

11 W.H. Press, B.P. Flannery, S.A. Teukolsky and W.T. Vetterling, Numerical Recipes, Cambridge University Press, Cambridge, 1989.

12 I. Blume, E. Smit, M. Wessling and C.A. Smolders, Diffusion in glassy and rubbery polymers, Makromol. Chem., Macromol. Symp., 45 (1991) 237-257.
13 K. Sommer, Correlation between primary chemical structure and property phenomena in polycondensates, Adv. Mater., (1991) 590.

14 A. Bos, Artificial Neural Networks as a Tool in Chemometrics, Ph.D. Thesis, University of Twente, Enschede, Netherlands, 1993. 